\title{
Activity of drugs against dormant Mycobacterium tuberculosis
}

\author{
A. Iacobino, G. Piccaro, F. Giannoni, A. Mustazzolu, L. Fattorini* \\ Istituto Superiore di Sanità, Department of Infectious, Parasitic and Immune-Mediated Diseases, Rome, Italy
}

\section{A R T I C L E I N F O}

Article history:

Received 15 September 2016

Accepted 21 September 2016

Available online $\mathrm{xxxx}$

\section{Keywords:}

Caseous granuloma

Cellular granuloma

Hydrophilic drugs

Lipophilic drugs

Mycobacterium tuberculosis

Tuberculosis

\begin{abstract}
A B S T R A C T
Objective/background: Heterogeneous mixtures of cellular and caseous granulomas coexist in the lungs of tuberculosis (TB) patients, with Mycobacterium tuberculosis (Mtb) existing from actively replicating (AR) to dormant, nonreplicating (NR) stages. Within cellular granulomas, the $\mathrm{pH}$ is estimated to be less than 6 , whereas in the necrotic centres of hypoxic, cholesterol/triacylglycerol-rich, caseous granulomas, the $\mathrm{pH}$ varies between 7.2 and 7.4. To combat TB, we should kill both AR and NR stages of Mtb. Dormant Mtb remodels lipids of its cell wall, and so lipophilic drugs may be active against NR Mtb living in caseous, lipid-rich, granulomas. Lipophilicity is expressed as logP, that is, the logarithm of the partition coefficient $(P)$ ratio $P_{\text {octanol }} / P_{\text {water }}$ In this study, the activity of lipophilic drugs $(\log P>0)$ and hydrophilic drugs $(\log \mathrm{P} \leqslant 0)$ against $\mathrm{AR}$ and NR Mtb was measured in hypoxic conditions under acidic and slightly alkaline pHs.

Methods: The activity of drugs was determined against AR Mtb (5-day-old aerobic cells: A5) and NR Mtb (12- and 19-day-old hypoxic cells: H12 and H19) in a Wayne dormancy model of Mtb H37Rv at pH 5.8, to mimic the environment of cellular granulomas. Furthermore, AR and NR bacilli were grown for 40 days in Wayne models at $\mathrm{pH} 6.6,7.0,7.4$, and 7.6, to set up conditions mimicking the caseous granulomas (hypoxia + slightly alkaline $\mathrm{pH}$ ), to measure drug activity against NR cells. Mtb viability was determined by colony-forming unit (CFU) counts.

Results: At pH 5.8, lipophilic drugs (rifampin, rifapentine, bedaquiline, PA-824, clofazimine, nitazoxanide: $\log P \geqslant 2.14$ ) reduced CFU of all cells (H12, H19, and A5) by $\geqslant 2 \log _{10}$. Among hydrophilic drugs (isoniazid, pyrazinamide, ethambutol, amikacin, moxifloxacin, metronidazole: $\log P \leqslant 0.01$ ), none reduced $\mathrm{H} 12$ and H19 CFUs by $\geqslant 2 \log _{10}$, with the exception of metronidazole. When Mtb was grown at different $\mathrm{pHs}$ the following Mtb growth was noted: at pH 6.6, AR cells grew fluently while NR cells grew less, with a CFU increase up to Day 15, followed by a drop to Day 40. AR and NR Mtb grown at pH 7.0, 7.4, and 7.6 showed up to 1 $\log _{10}$ CFU lower than their growth at $\mathrm{pH}$ 6.6. The $\mathrm{pHs}$ of all AR cultures tended to reach $\mathrm{pH}$ 7.2-7.4 on Day 40. The pHs of all NR cultures remained stable at their initial values $(6.6,7.0$, 7.4, and 7.6) up to Day 40. The activity of drugs against $\mathrm{H} 12$ and $\mathrm{H} 19$ cells was tested in hypoxic conditions at a slightly alkaline $\mathrm{pH}$. Under these conditions, some lipophilic drugs were more active ( $>5$ log CFU decrease after 21 days of exposure) against $\mathrm{H} 12$ and $\mathrm{H} 19$ cells than clofazimine, nitazoxanide, isoniazid, pyrazinamide, amikacin $(<1$ log CFU decrease after 21 days of exposure). Testing of other drugs is in progress.
\end{abstract}

\footnotetext{
* Corresponding author at: Istituto Superiore di Sanità, via Regina Elena 299, 00161 Rome, Italy.

E-mail address: lanfranco.fattorini@iss.it (L. Fattorini).

Peer review under responsibility of Asian African Society for Mycobacteriology.

http://dx.doi.org/10.1016/j.ijmyco.2016.09.061
} 


\section{Conflicts of interest}

Conclusion: Lipophilic drugs were more active than hydrophilic agents against dormant Mtb in hypoxic conditions at pH 5.8. The Wayne model under slightly alkaline conditions was set up, and in hypoxic conditions at a slightly alkaline $\mathrm{pH}$ some lipophilic drugs were more active than other drugs against NR Mtb. Overall, these models can be useful for testing drug activity against dormant Mtb under conditions mimicking the environments of cellular and caseous granulomas.

The authors have no conflicts of interest to declare. 